\title{
Usefulness of three-dimensional computed tomography of the larynx for evaluation of unilateral vocal fold paralysis before and after treatment: technique and clinical applications
}

\author{
Hiroyuki Hiramatsu • Ryoji Tokashiki • \\ Mamoru Suzuki
}

Received: 18 March 2007 / Accepted: 18 October 2007 / Published online: 10 November 2007

(C) Springer-Verlag 2007

\begin{abstract}
Laryngoplasty is well-known technique for unilateral vocal fold paralysis (UVFP). However, operation result are sometimes not as good as expected before surgery. Three-dimensional Computed tomography (3DCT) is useful for visualizing complicated intralaryngeal structures. Moreover, 3DCT is suited for analyzing the movement of the vocal fold and arytenoid cartilage because the technique is based on actual data from live patients. We have been used 3DCT of the Larynx for evaluation of UVFP before and after treatment. We uncovered some new findings about UVFP and reasons of unsatisfactory outcomes after operation. Technique and clinical applications of 3DCT for UVFP are outlined in this paper.
\end{abstract}

Keywords Three-dimensional computed tomography . Unilateral vocal fold paralysis · Thyroplasty

\section{Introduction}

The movement of the arytenoid cartilage is complicated [1, 2]. Three-dimensional computed tomography (3DCT) is useful for visualizing the location of the arytenoid cartilage. However, complete understanding of the laryngeal anatomy is necessarily to reconstruct the cartilages in three dimensions. In our institution, an otolaryngologist who specializes in the larynx utilizes 3DCT reconstruction. The use of

\footnotetext{
H. Hiramatsu $(\bowtie) \cdot R$. Tokashiki $\cdot$ M. Suzuki

Department of Otolaryngology,

Tokyo Medical University Hospital,

6-7-1, Shinjuku-ku, Nishishinjuku, Tokyo 160-0023, Japan

e-mail: hiro441741@aa.cyberhome.ne.jp
}

Windows ${ }^{\circledR}$ XP-compatible image-processing software enables various analyses in unilateral vocal fold paralysis. This study reports the clinical application of 3DCT in the laryngeal region using the image-processing software Mimics (Materialise, Yokohama, Japan).

\section{Production of 3DCT}

Computed tomography was performed in the horizontal plane using a CT scanner (Sensation Cardiac 64; Siemens, Munich, Germany) with a $1 \mathrm{~mm}$ slice width, a helical pitch of 1, and an image production interval of $0.5 \mathrm{~mm}$. Several other studies have used slice widths of around $1 \mathrm{~mm}[3-6]$.

Scans were made when the glottis was closed (with phonation) and open. Each patient was scanned for about $2 \mathrm{~s}$. The speed of the scan enables scanning of patients with unilateral vocal fold paralysis during a brief phonation and minimizes motion artifact. Before scanning, doctors (the authors) were consulting the patient about the timing between phonation and the scan.

The data were transferred using the Dicom system. Mimics ver. 9.01 (Materialise) was used to create 3DCT images through its surface-rendering protocols. Using Windows $^{\circledR}$ XP $\left(\right.$ Microsoft $^{\circledR}$, Redmond, WA, USA), 3DCT images can be shown in axial, coronal, sagittal, and 3D display windows (Fig. 1).

The threshold was set from 69 to $85 \mathrm{HU}$ for the thyroid and cricoid cartilages and 52 to $69 \mathrm{HU}$ for the arytenoid cartilage. Visualization of the vocal process is usually limited to its base because of its elastic cartilage. In addition, the laryngeal lumen was visualized. The threshold was set between -600 and $-100 \mathrm{HU}$ [7]. 
Fig. 1 Actual view seen with the Mimics image-processing software. Using Windows ${ }^{\circledR} \mathrm{XP}$ (Microsoft $^{\circledR}$ ), 3DCT images can be created with axial, coronal, sagittal, and 3D display windows. The use of Windows XP-compatible image-processing software enables various analyses in unilateral vocal fold paralysis

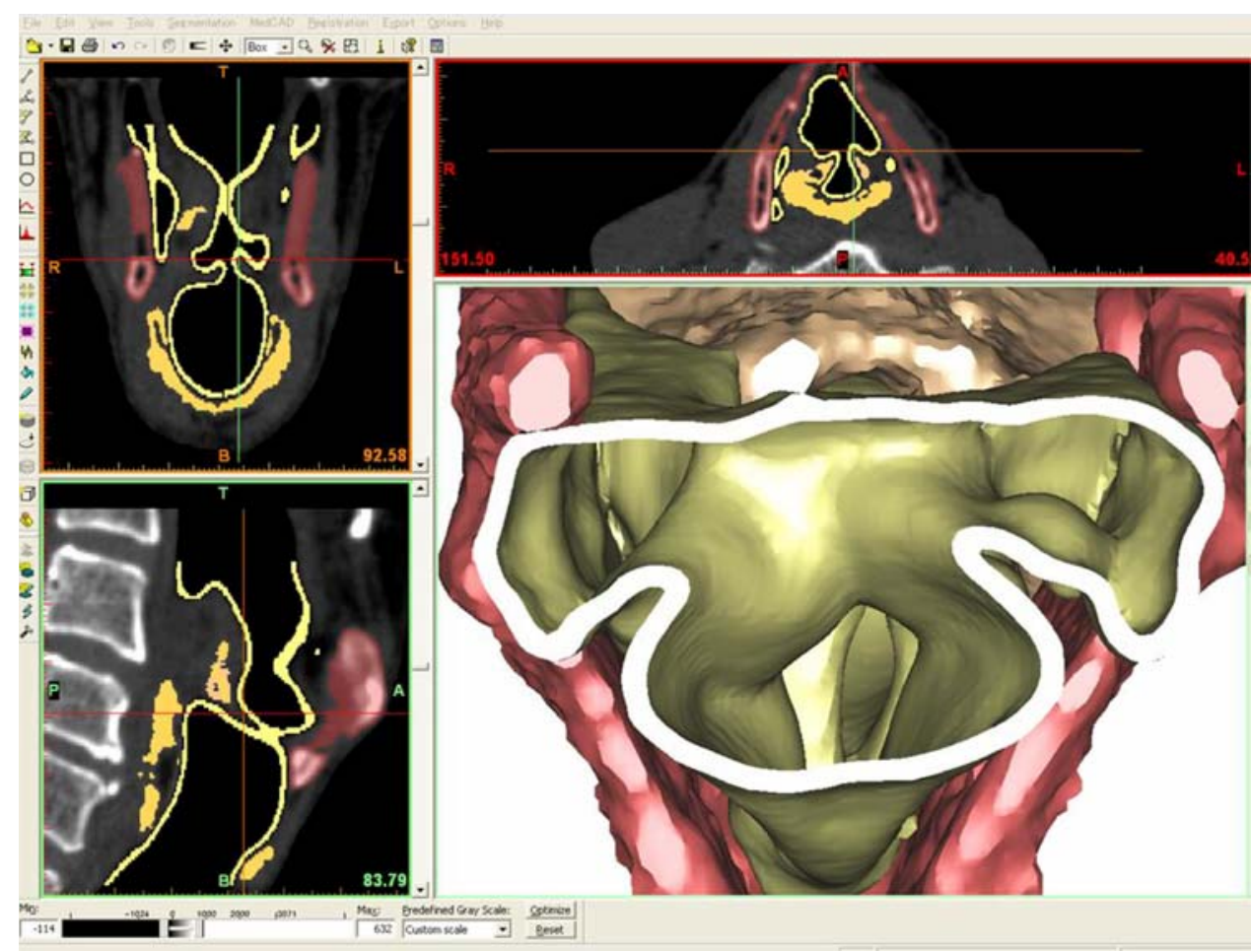

\section{Stereoscopic location of the arytenoid cartilage in unilateral vocal fold paralysis}

First, we present a case of severe unilateral vocal fold paralysis with a mean flow rate (MFR) of $1,200 \mathrm{ml} / \mathrm{s}$ (Fig. 2). The patient was an 80-year-old man suffering from paralysis following aortic aneurysm surgery.

During inspiration, the arytenoid cartilage on the healthy side was located in the lateral part of the cricoarytenoid joint. Simultaneously, the vocal process was located outside and above the joint. In comparison, the arytenoid cartilage on the paralyzed side was located in the dorsocranial part of the cricoarytenoid joint, while the paralyzed vocal process was located inferiorly and the arytenoid cartilage dropped forward. This position on the paralyzed side was the result of posterior cricoarytenoid muscle paralysis.

During phonation, the healthy side rocked and moved to the ventromediocaudal part of the cricoarytenoid joint to adduct the vocal cord, and the vocal process moved inward and downward. Conversely, the paralyzed side glided dorsocranially in comparison with its position during inspiration. Therefore, the arytenoid cartilage showed a rocking movement on the healthy side, and a gliding movement on the paralyzed side. This gliding movement on the paralyzed side was a passive movement. the paralyzed arytenoid underwent passive movement caused by contact with the mobile side during phonation. The vocal fold blown up may affect this passive movement. We call such arytenoids "flaccid arytenoids." The position of the paralyzed arytenoid depends on the severity of paralysis.

\section{Surgical simulation}

This section describes type I thyroplasty. It is important to determine the level of the vocal folds in this procedure. Note that the arytenoid cartilage on the paralyzed side glides dorsocranially, as mentioned above. The paralyzed vocal fold becomes higher than the vocal fold on the healthy side during phonation. The type I thyroplasty window should be based on the vocal cord level on the healthy side during phonation. Therefore, the vocal fold level of the healthy side must be projected onto the thyroid cartilage of the paralyzed side.

Figure 3 compares the upper surface of the vocal fold between our 3DCT simulation and the original type I procedure reported by Isshiki et al. [8]. The figure shows that the upper surface level of the vocal fold in the original procedure is consistent with the vocal fold level on the paralyzed side detected by 3DCT. This might have arisen because the upper surface level of the vocal cord in the original procedure was based on cadaveric studies of the larynx. However, the vocal process on the healthy side during phonation is lower than that on the paralyzed side. Therefore, the design should be made consistent with the healthy side. Individualized simulation for each case is needed because of the shape difference between left and right laminas of the thyroid cartilage (especially the laryn- 
Fig. 2 The locations of the arytenoids in severe unilateral vocal fold paralysis. During inspiration, the healthy side (yellow) was located in the lateral part of the cricoarytenoid joint, while the paralyzed side ( $r e d$ ) was located in the dorsocranial part of the cricoarytenoid joint. During phonation, the healthy side rocked and moved to the ventromediocaudal part of the cricoarytenoid joint, while the paralyzed side glided dorsocranially. This movement is a passive movement
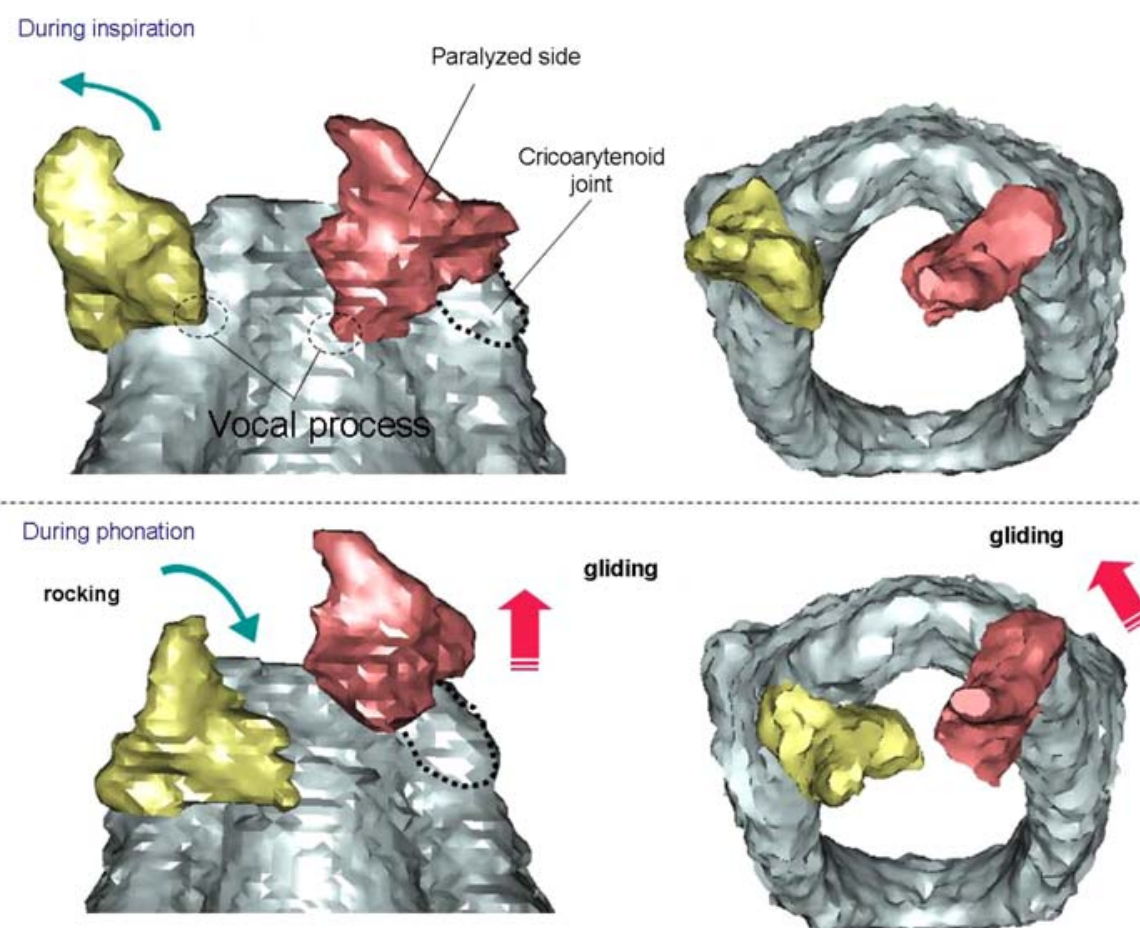

gliding

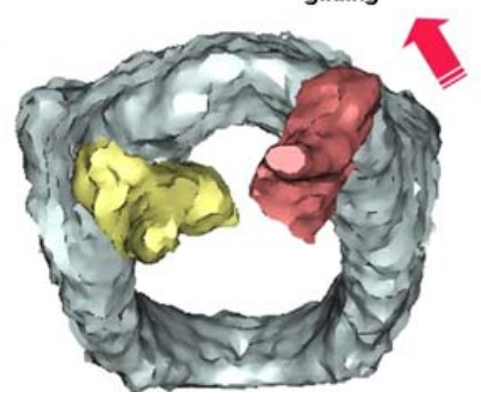

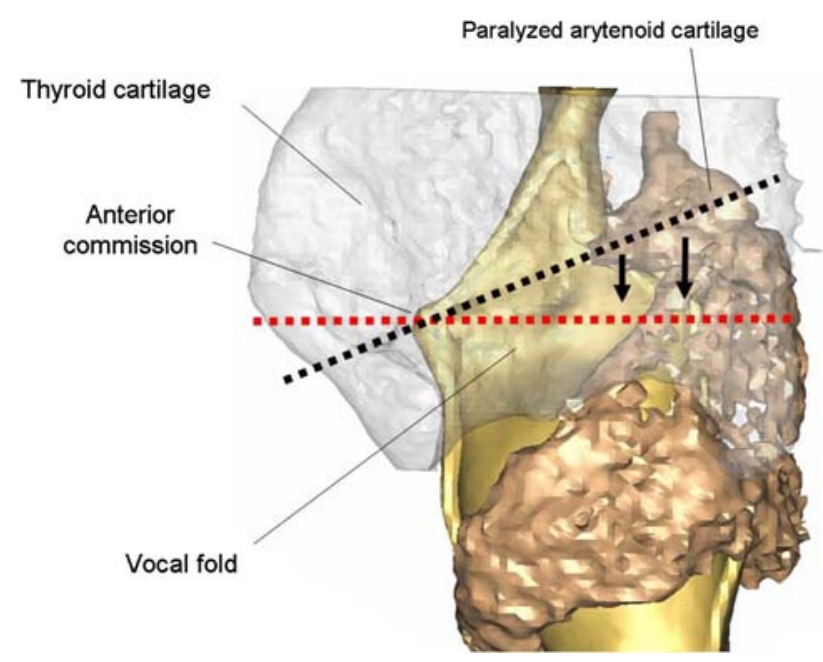

Fig. 3 A comparison of the upper surface of the vocal fold between the 3DCT simulation (red dotted line) and the original type I procedure reported by Isshiki (black dotted line). The figure shows a 3D image processed to make the thyroid cartilage more radiolucent, allowing the healthy vocal fold to be visualized. The upper surface level of the vocal fold with the original procedure is consistent with the vocal fold level of the paralyzed side detected by 3DCT. However, the vocal process of the healthy side during phonation is located below than that of the paralyzed side

geal deviation) $[9,10]$. The program Mimics allows distances to be measured on a personal computer; for example, the distances from the inferior notch or inferior nodule to the surface of the vocal fold may be measured. The 3DCT could contribute to a better oriented and designed thyroplasty window.

\section{Postoperative evaluation}

Case 1

A 69-year-old woman underwent arytenoid adduction for right recurrent nerve paralysis after thyroid carcinoma surgery. According to the postoperative 3DCT images (Fig. 4), the vocal process adducted to the appropriate position. However, a large glottal chink was observed during phonation. In this case, the cricothyroid joint was released, and a thread placed on the muscular process was fixed to the anterior part of the thyroid cartilage. Since the cricothyroid joint was released, the cricoid cartilage (arytenoid cartilage) and thyroid cartilage were close together, which shortened the anteroposterior diameter of the vocal fold. The vocal fold relaxed and lost its tension.

\section{Case 2}

A 64-year-old man underwent type I thyroplasty for left recurrent nerve paralysis after esophageal cancer surgery (Fig. 5). This patient had relatively severe paralysis, but still underwent a type I thyroplasty only. The postoperative 3DCT image shows that the Gore-Tex ${ }^{\circledR}$ is inserted at the proper level of the vocal fold. However, the posterior parts of the vocal fold were at different levels because the flaccid paralyzed arytenoid cartilage was blown toward the dorsocranial part of the cricoarytenoid joint, as mentioned above. The MFR was improved from $770 \mathrm{ml} / \mathrm{s}$ preoperatively to 
Fig. 4 Case 1: A 69-year-old woman underwent arytenoid adduction for right recurrent nerve paralysis after thyroid carcinoma surgery. a In the postoperative 3DCT images, the vocal process appears to adduct to the appropriate position. b A large glottal chink is observed during phonation. c Since the cricothyroid joint is released, the cricoid (arytenoid cartilage) and thyroid cartilages are close together. d The anteroposterior diameter of the vocal fold (double-headed arrow) is shortened. The vocal fold relaxes and loses its tension
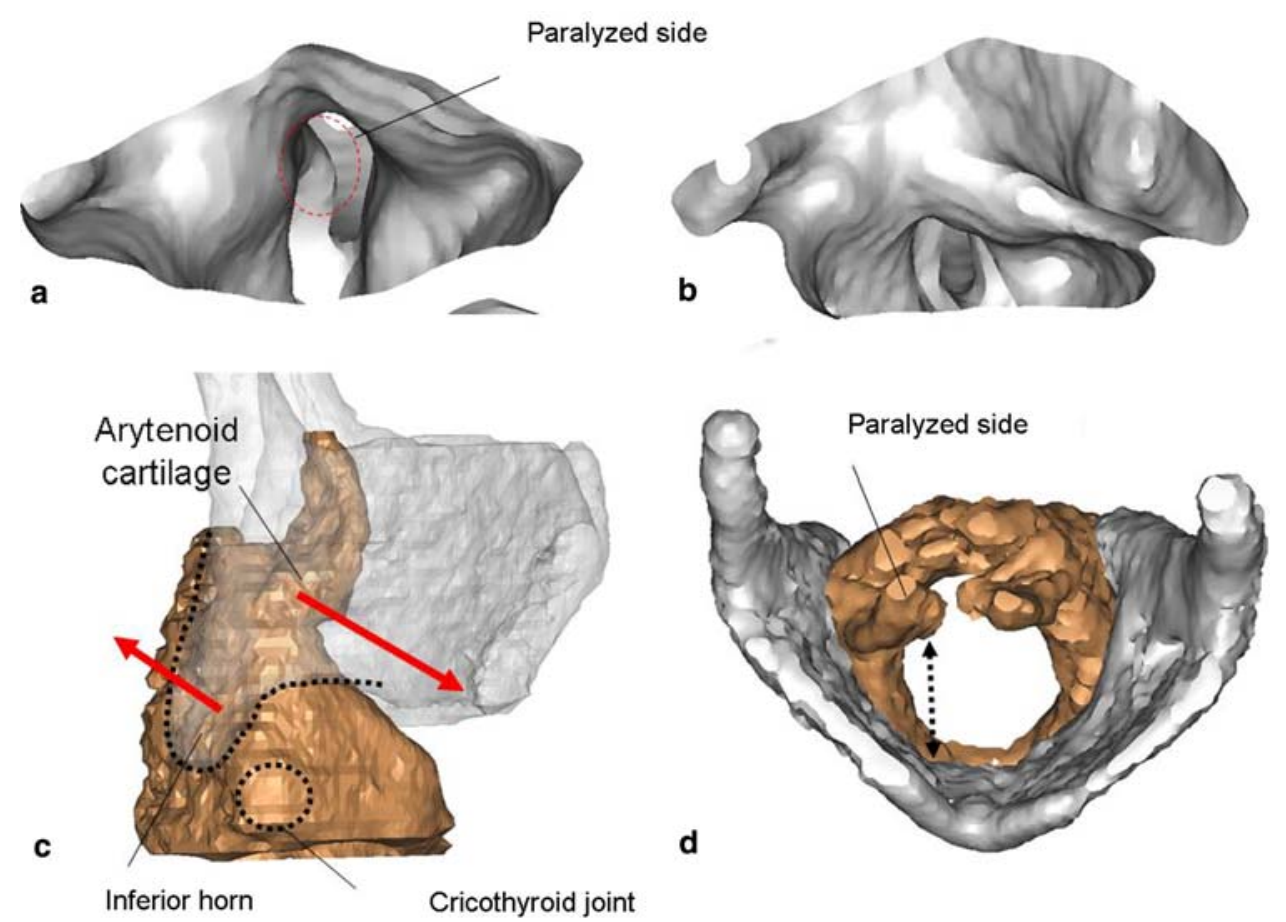

a

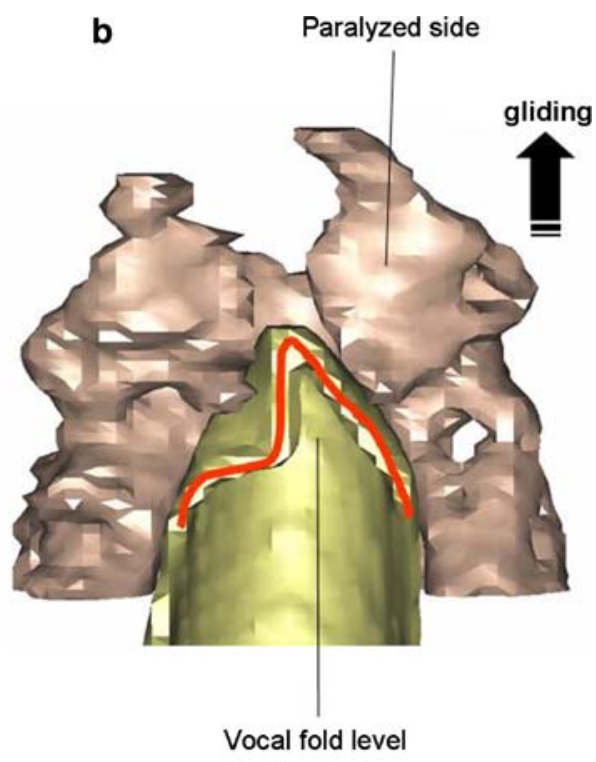

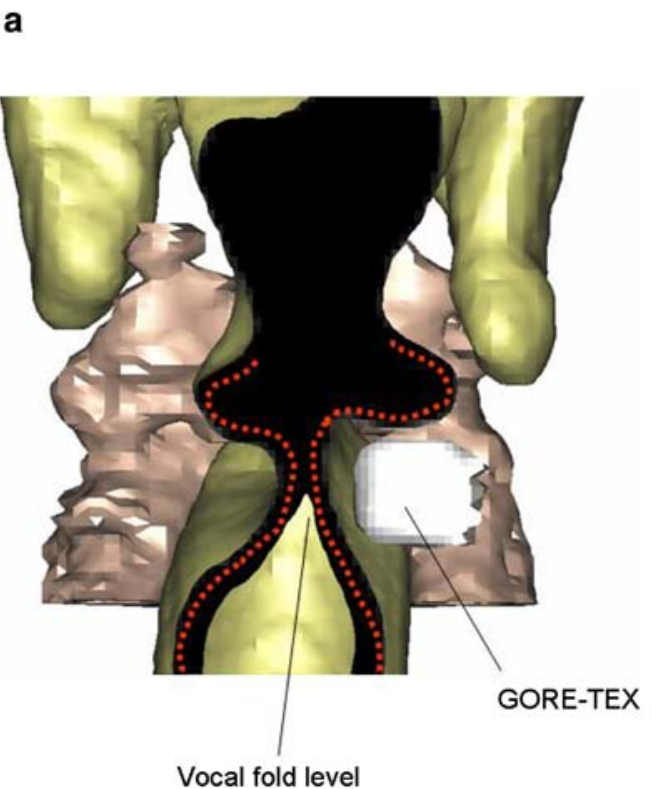

b

Paralyzed side
Fig. 5 Case 2: A 64-year-old man underwent type I thyroplasty for left recurrent nerve paralysis after esophageal cancer surgery. a The postoperative 3DCT image shows that the Gore-Tex ${ }^{\circledR}$ was inserted at the proper level of the vocal fold. b A level difference is seen in the posterior part of the vocal fold because the flaccid paralyzed arytenoid cartilage was blown toward the dorsocranial part of the cricoarytenoid joint during phonation
$290 \mathrm{ml} / \mathrm{s}$ postoperatively. If the posterior support of the vocal fold had been fixed, the vocal fold would have developed tension against the flow of expired air during phonation, further improving the voice.

\section{Case 3}

A 70-year-old man was given a silicon injection 20 years earlier to treat left recurrent nerve paralysis after thoracic aortic aneurysm surgery (Fig. 6). The MFR was $650 \mathrm{ml} / \mathrm{s}$, which improved insufficiently. The 3DCT showed that the silicon was injected widely from the anterior to posterior parts of the vocal fold. Silicon is also injected near the arytenoid cartilage. It was thought that the original surgeon had intended to adduct the arytenoid cartilage. However, the arytenoid cartilage glides dorsocranially, increasing the vertical difference in the level of the posterior part of the vocal fold. The vocal process was not adducted and the upper part of the arytenoid cartilage moved medially in an unnatural manner. As described earlier, during phonation, the arytenoid cartilage on the healthy side moves by rocking to the ventromediocaudal 
Fig. 6 Case 3: A 70-year-old man underwent a silicon injection 20 years earlier to treat left recurrent nerve paralysis after thoracic aortic aneurysm surgery. a The 3DCT shows that the silicon is injected widely from the anterior to posterior parts of the vocal fold. b Silicon is also injected near the arytenoid cartilage. The arytenoid cartilage glides dorsocranially; the vocal process is not adducted and the upper part of the arytenoid cartilage is unnaturally shifted medially. The vertical difference in the level of the posterior part of the vocal fold increased. $\mathbf{c}$ The effect of the injection on posterior vocal fold closure is thought to be due mainly to the volume increase in the posterior part of the vocal fold
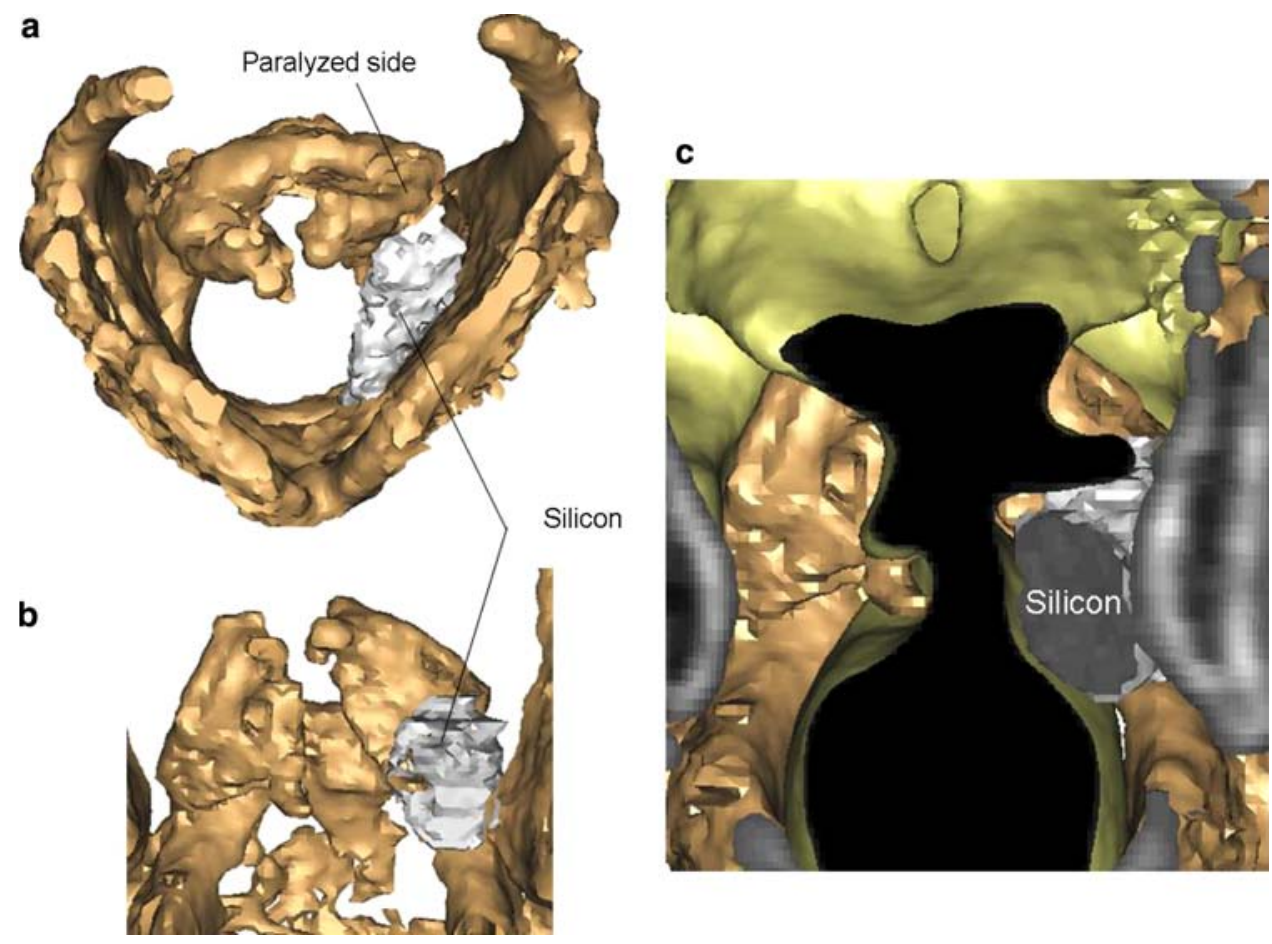

Fig. 7 Case 4: A 77-year-old man underwent fat injection for left recurrent nerve paralysis after esophageal cancer surgery. a The 3DCT (CT endoscopic image) suggests that the vocal cord closure is sufficient. b The lateral view shows fat remaining 3 years after the injection, which seems to be pushing the entire vocal fold. c The coronal view revealed that the difference in the levels of the vocal fold had not improved

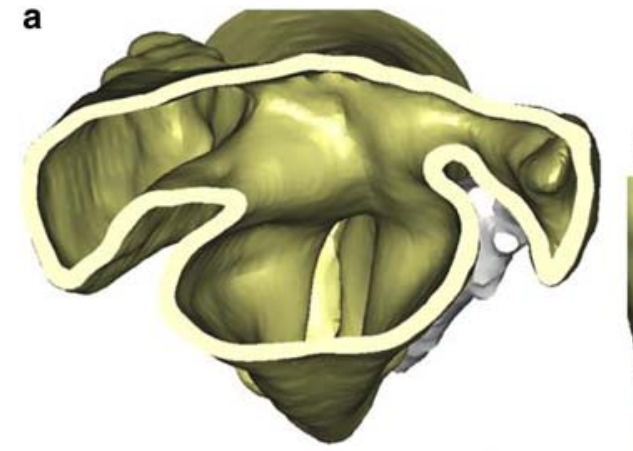

b

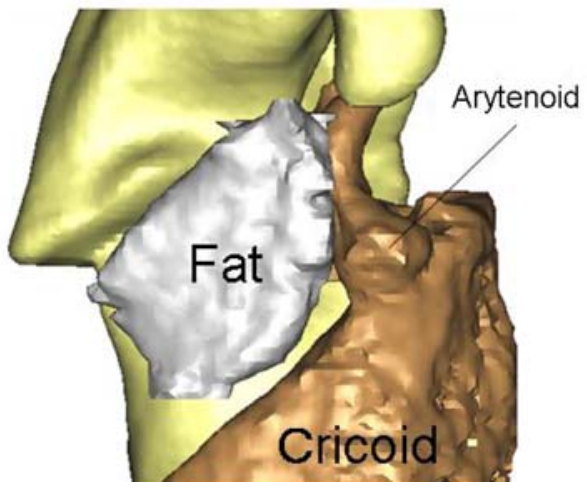

C

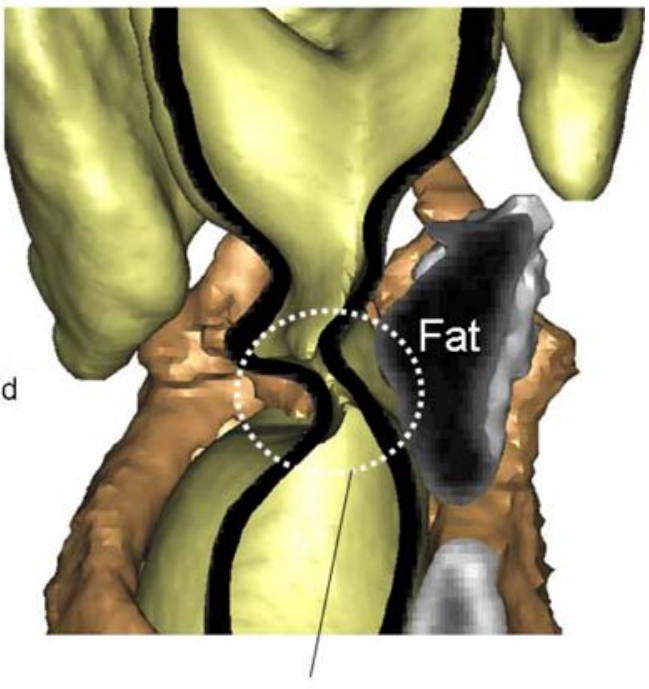

Vocal fold level part of the cricoarytenoid joint, and the vocal process is moved inward. Arytenoid adduction [10,11] is a procedure that resolves the level difference between the two vocal fold by reproducing the rocking motion of the paralyzed arytenoid cartilage. In contrast, injection and type I surgery are techniques to medialize the vocal fold by increasing its volume. An injection near the arytenoid cartilage moves the arytenoids to a dorsocranial position, and they never adduct naturally. If arytenoid adduction is scheduled after these techniques, the injected materials could interfere with the second procedure. The effect of the injection on posterior vocal fold closure arises mainly 
from the increased volume in the posterior part of the vocal fold.

\section{Case 4}

A 77-year-old man received a fat injection for left recurrent nerve paralysis after esophageal cancer surgery. Three years after the fat injection, the maximum phonation time improved from $3 \mathrm{~s}$ before the injection to $7 \mathrm{~s}$ after the injection. The 3DCT image indicates that the vocal cord closure was sufficient (Fig. 7a). In the lateral view, remaining fat is seen 3 years after the injection and it appears to be pushing the entire vocal fold (Fig. 7b). However, the coronal section CT image revealed that the level difference of the two vocal folds had not improved. The vocal folds overlapped so that the incomplete closure was resolved (Fig. 7c). Fat, unlike silicon, may not interfere with the vocal fold vibration of the healthy side, even if the vocal cords overlap, because fat is soft.

\section{Conclusions}

Laryngeal 3DCT is useful for determining the stereoscopic configuration of the arytenoid cartilage. In addition, 3DCT enables observation of the laryngeal framework from every angle, and is also useful for surgical simulation and feedback after surgery.

\section{References}

1. Von Leden H, Moore P (1961) The mechanics of the cricoarytenoid joint. Arch Otolaryngol 73:63-72

2. Pernkopf E (1980) In: Atlas of topographical and applied human anatomy. Head and Neck Vol. 1, Larynx, Urban and Schwarzenberg, Baltimore, pp 313-319

3. Meglin AJ, Biedlingmaier JF, Mirvis SE (1991) Three-dimensional computerized tomography in the evaluation of laryngeal injury. Laryngoscope 101:202-207

4. Rubin JS, Summers P, Harris T (1998) Visualization of the human larynx: a three-dimensional computer modeling tool. Auris Nasus Larynx 25:303-308

5. Fried MP, Moharir VM, Shinmoto H et al (1999) Virtual laryngoscopy. Ann Otol Rhinol Laryngol 108:221-226

6. Walshe P, Hamilton S, McShane D, McConn Walsh R, Walsh MA, Timon C (2002) The potential of virtual laryngoscopy in the assessment of vocal cord lesions. Clin Otolaryngol 27:98-100

7. Yumoto E, Sanuki T, Hyodo M, Yasuhara Y, Ochi T (1997) Three-dimensional endoscopic mode for observation of laryngeal structures by helical computed tomography. Laryngoscope 107:1530-1537

8. Isshiki N, Okamura H, Ishikawa T (1975) Thyroplasty type I (lateral compression) for dysphonia due to vocal cord paralysis or atrophy. Acta Otolaryngol 80:465-473

9. Hiramatsu H, Yamaguchi H, Niimi S et al (2004) An attempt for construction of precision three-dimensional laryngeal model (in Japanese). Nippon Jibiinkoka Gakkai Kaiho 107:945-955

10. Hiramatsu H, Tokashiki R, Yamaguchi H et al (2006) Threedimensional laryngeal model for planning of laryngeal framework surgery. Acta Otolaryngol 126:515-520

11. Tokashiki R, Hiramatsu H, Tsukahara K, et al. (2007) A new procedure of arytenoid adduction combined with type I thyroplasty under general anesthesia using a laryngeal mask. Acta Otolaryngol 127:328-331 